\title{
Computer Simulation of Single-Ion Trajectories in Paul-Type Ion Traps
}

\author{
Frank A. Londry and Roland L. Alfred \\ Department of Physics, Trent University, Peterborough, Ontario, Canada
}

\author{
Raymond E. March \\ Department of Chemistry. Trent University. Peterborough, Ontario, Canada
}

\begin{abstract}
The computer simulation of single-ion trajectories using a number of computer programs is described together with associated theory. The programs permit calculation of ion trajectories while the ion is subjected to collisions with buffer gas of variable pressure, resonance excitation in any of three modes, and static or ramped $D C$ and radiofrequency levels. Initially, the programs were designed for the calculation of ion trajectories in a quadrupole ion trap. The programs now permit such calculations for ions confined in traps having electrodes shaped to include percentages of hexapole and octupole components in the electric field as well as electrode surface geometries for which there is no closed-form expression. The Langevin collision theory is reviewed and a theoretical treatment of the multipole trap is presented. (] Am Soc Mass Spectrom 1993, 4, 687-705)
\end{abstract}

$\mathrm{N}$ umerical methods were first applied to the analysis of single-ion trajectories in the quadrupole ion trap some 25 years ago by Dawson and Whetten [1]. These early studies were extended [2] to an examination of ion ejection in which a range of ion masses were confined simultaneously. The application of Monte Carlo methods to the investigation of ion motion indicated that ions migrate to the center of the ion trap with an accompanying diminution in ion kinetic energy, and from which they can be extracted with increased efficiency $[3,4]$. The use of Monte Carlo methods was extended to the study of an ensemble of two ion species [5].

André and Schermann [6] studied the spatial and velocity distribution of an ion population stored in an ion trap and subjected to collisions with rare gas atoms. $\Lambda$ mathematical formalism of temporal invariance, developed by Vedel et al. [7], has been applied to the study of the statistical spatial and energy distributions of trapped ions subjected to collisional cooling. These studies were extended to the space charge case by Vedel and André [8,9].

Representative trajectories for trapped ions of three different masses, with working points on the $q_{z}$ axis and in a collision-free and resonance-free system, have been calculated by Louris et al. [10] by integration of the Mathieu equation. Lunney et al. [11] have applied finite element analysis to the calculation of fields within

Address reprint requests to Raymond E. March, Department of Chemistry, Trent University, Peterborough, Ontario, K9J 7B8, Canada. the ion trap from which trajectories were calculated. A field modeling process, in which the field was reconstructed using a summation of multipole potentials, has been developed by Lunney and Moore [12].

Previously unpublished trajectory plots by Bexon and Todd and by Fies, Jr., for ions stored within the ion trap and subjected to collisions with helium buffer gas, appeared in a publication by Todd [13]. Bexon and Todd calculated one-dimensional (axial) trajectories of $m / z 25$ ions showing the effect of regular collisions with helium atoms; the motion of $m / z 69$ ions in the $r-z$ space plane both with and without helium buffer gas was calculated by Fies, Jr.

Pedder and Yost [14] have developed the HYPERION program for the calculation of ion trajectories under the influence of resonant excitation; this program uses a modified form of the Mathie1 equation together with a fourth-order Runge-Kutta algorithm. A parallel capacitor model was used for dipolar excitation; the simulated final kinetic energy as a function of tickle frequency at constant fluence compared well with experimental loss in parent ion intensity as a function of tickle frequency.

An ion trap simulation program (ITSIM) has been used with great success by Cooks and co-workers [15]. The features of the program permit simulation of external ion injection, bath gas dampening, and resonance excitation. The equations used to calculate an ion's trajectory have a modified form of the "conventional equations of ion motion" for an ion trap. Plots of unperturbed ion motion and ion axial ejection by reso- 
nance excitation have appeared recently [16]. Two further simulations have been carried out with ITSTM: first, rapid radial excitation to instability upon the application of a short-duration, high-voltage DC pulse to the end-cap electrodes [17] is compatible with the observed fragmentation pattern induced by collisions with a surface (ring electrode); and second, calculated ion kinetic energies upon ejection agree well with experimental values [18]. The limitations of the singleion simulation method, together with the dramatic improvements in computer speed, prompted Cooks and co-workers $[19,20]$ to develop multiparticle simulations using a Numerical Quadrupole Simulation (NQS) program. A copy of the revised ITSIM program [20] has been made available to the research community.

A Taylor-McLaurin expansion in the solution of the trajectory equations, used in the phase space formalism, has been proposed for the calculation of ion trajectories in the ion trap [21]; however, no example of this method has been developed thus far.

Single-ion simulations have been carried out by Franzen and co-workers $[22,23]$ to investigate the performance of ion traps constructed with nonlinear fields superimposed on the main quadrupole field. Time-of-flight simulation studies of $\mathrm{Xe}^{+}$have been carried out by Vedel and Vedel [24] and, more recently from the same laboratory, by Rebatel [25].

Some 4 ycars ago, researchers at the universities of Trent, Kent, and Provence set out to develop a microcomputer program for the calculation of the trajectories of individual ions subjected to resonance radiation while stored in a quadrupole ion trap of ideal geometry. Concurrent with these simulation studies, experimental studies were carried out at Kent and Provence on resonance excitation and the radiofrequency (RF) absorption spectra of trapped ions, respectively [26].

As the motion of ions stored in a quadrupole ion trap is characterized by a frequency spectrum, irradiation of ions at a frequency component of this spectrum can lead to excitation of translational motion as the ions absorb power from the field due to the resonance irradiation. Indeed, resonant absorption of power was the method employed by Paul et al. [27] and Fischer [28] for detection of trapped ions. With the onset of commercial ion traps, resonance excitation of ions stored in a quadrupole ion trap [29] is now employed on a wide scale. The supplementary RF potential oscillating at a selected frequency has been referred to as an auxiliary, or "tickle," voltage as opposed to the main RF potential, which is known as the "drive" potential.

Resonance excitation can be used to enhance stored ion kinetic energy or to eject ions from an ion trap. In the former case, resonance excitation leading to enhancement of ion kinetic energy can be used to open endothermic ion-molecule reaction pathways to form product fragment ions or product associative ions; alternatively, ion kinetic energy can be transferred to internal energy and accumulated in the resonantly excited species through collisions with a helium buffer gas to the point of ion fragmentation. Collision induced dissociation wrought by resonance excitation in this way is a near-essential element of tandem mass spectrometry operation of the ion trap. In the latter case, ion ejection can be accomplished using a specific tickle frequency while the RF drive amplitude is held constant so that ions of a specific $m / z$ are ejected. Alternatively, the RF drive amplitude can be ramped while a tickle voltage is applied so that several or all ion species are ejected mass-selectively as each species comes into resonance with the chosen tickle frequency. In this RF scanning mode, the mass range of the ion trap can be increased by an order of magnitude or so [30].

Clearly then, it was of some interest to develop a simulation program with which it would be possible to explore the effects of resonance and near-resonance excitation of single ions stored in a quadrupole ion trap and, where possible, to compare the behavior of a collectivity of single ions with experimental observations of an ensemble of ions.

Ion trajectories may be calculated readily for single ions stored in an ideal quadrupole ion trap in the resonance-free situation using the solution to the Mathieu equation. The initial program designed to perform these calculations was designated MA; this program, which uses the exact solution to the Mathieu equation, was used as a basis for comparison with subsequent programs by which the effects of resonance excitation could be explored but in which the tickle voltage had been reduced to zero.

Resonance excitation may be induced by any of three arrangements for applying an auxiliary potential to ion trap electrodes and, herein, the first major problem was encountered. While for resonance excitation in-phase potentials may be applied to each end-cap electrode (quadrupolar), or potentials $180^{\circ}$ out of phase may be applied to each end-cap electrode (dipolar), or a potential may be applied to one end-cap electrode while the other is grounded (monopolar), only for the quadrupolar mode can the second-order differential equations of motion be written in closed form. The monopolar mode had been employed experimentally earlier and the dipolar mode was employed in the commercial ion trap, but the quadrupolar mode had not been used at all. Nevertheless, as the quadrupolar arrangement was the most simple arrangement to simulate, the first simulation study of resonance excitation was undertaken using a simulation program for quadrupolar resonance (SPQR). The results of the first study were presented in two papers [31, 32]. A comparison of the SPQR program with the MA program (with the tickle voltage amplitude of the SPQR program set to zero) is presented later.

To tackle the simulation of the monopolar and dipo- 
lar arrangements, it was necessary to develop a third program using the field interpolation method (FIM). The development of FIM constituted a considerably greater task, but, in view of the interest in applying the dipolar arrangement particularly, the task was undertaken. In retrospect, we believe that this decision was well justified. It is now possible to apply FIM (with or without resonance excitation) to the simulation of ion trajectories in the commercial ion trap (in which the inter-electrode spacing exceeds that for the ideal case and the ion trap electrodes are no longer equipotential surfaces of a quadrupole field), in multipole ion traps (i.e., ion traps whose electrodes have been shaped to include a certain percentage of hexapole and octupole components in the electric field inside the trap), and also in ion traps whose electrode surface geometries follow no closed-form expression and must be represented numerically.

Although initially developed as two separate programs, SPQR and FIM shared so many of the same features, such as the ability to take account of collisions of the stored ion with helium buffer gas, that they were combined, recenfly, into an integrated system for ion simulations, or ISIS. ISIS uses the solver routine (simulator) best suited to the electrode configuration and mode of operation specified at run-time.

In the discussion that follows, a comparison of trajectories calculated by the different simulators is presented, followed by an overview of those aspects of the system operation that apply equally to all three simulators. Then the operation of each simulator is described in detail. Throughout the discussion, examples of calculated ion trajectories are presented to illustrate ion behavior in a number of different situations. Finally, the Langevin collision theory is reviewed in Appendix $A$ and a theoretical treatment of the multipole ion trap is presented in Appendix B.

\section{Inter-Comparison of Simulators}

\section{A Comparison of FIM with the Mathieu Analytical Solution.}

Ion trajectories calculated by FIM for the tickle-free situation can be compared directly with those calculated using the analytic solution of the Mathieu equation. An exacting comparison of the calculated trajectories can be made by superimposing the respective temporal variations of ion kinetic energy, radial position, and axial positions calculated by each method for a designated interval of time. In Figure $1 \mathrm{a}-\mathrm{c}$ are shown the respective superimpositions of the temporal variations for an ion of $m / z 134$ for a duration of $10 \mu \mathrm{s}$, that is, $10^{4}$ time-steps by each method of calculation. The agreement between the two methods for the temporal variation of both ion kinetic energy (Figure la) and axial position (Figure 1c) is excellent in that the
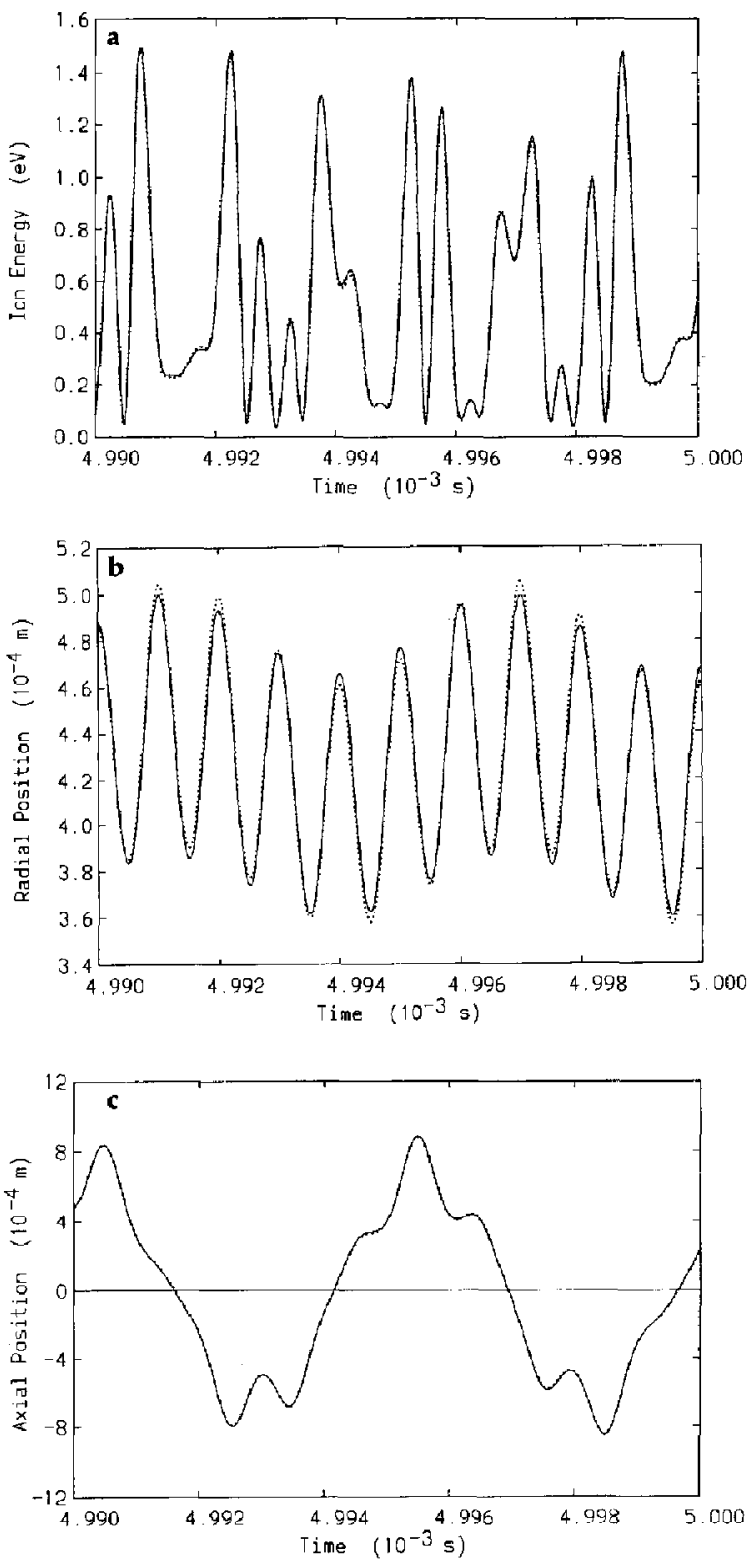

Figure 1. The trajectory of $m / z 134$ calculated for the nonresonant case using FIM $(\cdots)$, with an interpolation of order 3 , for a period of $5 \mathrm{~ms}$ using time steps of $1 \mathrm{~ns}$. The working point was $\left(a_{z}, q_{z}\right)=(0,0.5), r_{0}=10 \mathrm{~mm}$, drive frequency $=1 \mathrm{MHz}$. The trajectory was calculated also by using the Mathieu analytic solution (- $\longrightarrow$. Temporal variations of: (a) kinetic energy; (b) radial position; (c) axial position during the final $10 \mu s$ of the calculated trajectories.

temporal variations of these trajectory parameters are virtually identical. While minor disparities are seen in the radial displacement maxima and minima (Figure $1 b$ ), the agreement is quite remarkable when it is considered that the comparison covers the final $10^{4}$ 
time-steps of a calculation that spanned a total of $5 \times 10^{6}$ time-steps $(5 \mathrm{~ms})$.

When the time-step is increased from $1 \mathrm{~ns}$ to $5 \mathrm{~ns}$, disparities in temporal variations become more evident toward the end of the simulation period.

\section{Comparison of FIM with SPQR}

The performance of FIM in calculating trajectories of ions subjected to quadrupolar excitation can be compared directly with that of SPQR. The criteria used for comparison here are, again, the temporal variation of ion kinetic energy, radial and axial excursions together with the total irradiation period required for ion ejection. Trajectories of an ion of $m / z 134$ for a total of six different irradiation situations were examined by both FIM and SPQR using time-steps of 5 ns.

Ion trajectories were calculated for each of three working points $\mathrm{A}, \mathrm{B}$, and $\mathrm{C}$ on the $q_{z}$ axis $\left(\mathrm{A}, a_{2}=0\right.$, $q_{z}=0.4997 ; \mathrm{B}, q_{z}=0.3750 ; \mathrm{C}, q_{z}=0.2502$ ). In each case the ion of $m / z \quad 134$ was irradiated in the quadrupolar mode with an auxiliary potential of amplitude $10 \mathrm{~V}_{(0-p)}$ at a frequency $\beta_{z} \Omega / 2$. The simulations carried out by FIM and SPQR each indicated that identical irradiation periods of $1.248 \times 10^{-4} \mathrm{~s}, 7.387 \times$ $10^{-5} \mathrm{~s}$, and $4.580 \times 10^{-5} \mathrm{~s}$ were required to bring about ion ejection at the working points $A, B$, and $C$, respectively. When the amplitude of the auxiliary potential was reduced to $3 \mathrm{~V}_{(0-p)}$, again identical irradiation periods were indicated by the two methods for ion ejection at the working points $B$ and $C$; the irradiation periods indicated were $2.959 \times 10^{-4} \mathrm{~s}$ and $1.468 \times$ $10^{-4} \mathrm{~s}$, respectively. The working point $A$, that is, with the highest value of $q_{z}$, was not examined. When the auxiliary potential amplitude was reduced to $1 \mathrm{~V}_{(0-\mathrm{p}) \text { s }}$ the working point $\mathrm{C}$ only was examined. Once again, excellent agreement between FIM and SPQR was obtained in that both methods predicted irradiation periods of $4.710 \times 10^{-4} \mathrm{~s}$ for ion ejection.

Thus, FIM and SPQR indicated identical irradiation periods to ejection for each of the six irradiation conditions examined. Furthermore, the temporal variations of ion position and kinetic energy as obtained by FIM and SPQR were in good agreement. While better agreement among these criteria could be achieved with shorter time-steps, for example, 1 ns, the duration of each trajectory calculation with the shorter time-step became excessively long. FTM reproduced the striking feature of earlier simulations carried out using SPQR [33] in that radial ejection of the subject ion was indicated when radiation at the fundamental axial secular frequency, $\beta_{z}$, was employed.

A number of comparisons have been made of the results of simulations with experimental data. A comparison of the relative magnitudes of absorption coefficients for both dipolar and quadrupolar resonant excitation yielded quite satisfactory agreement [33], whereas for the comparison of the induction fluence required for ion ejection, agreement was somewhat disappointing [34]. These comparisons were made with experimental data obtained on a homemade ion trap at the University of Provence and a commercial device at the University of Kent. Only later did it become apparent that the ion traps in these two locations were much more dissimilar than we thought they were.

Around the time of these experiments we became aware of the ion traps developed by Jochen Franzen; the fields in these traps differ from that of the pure quadrupole ion trap in that they contain hexapole and actupole components. As such ion traps piqued our interest, the simulation program was modified yet again so that trajectories could be calculated for ions confined in these traps. No sooner was our program completed than it was learned that the commercial ion trap was "stretched," in that the separation between the end-cap electrodes was greater than that for the pure quadrupole ion trap [35]. In the light of this announcement, we have pointed out [36] that the stretching of the ion trap will introduce multipoles which can affect absorption peak shape and the induction fluence required for ion ejection. Induction fluences obtained by simulation studies of the ideal quadrupole ion trap are approximately one-third of those required by an ion trap mass spectrometer (ITMS). The longer irradiation times required with the ITMS may be due to the off-resonance drift as ions experience the nonlinear forces within the trapping field and, perhaps, to a deeper potential-well depth in the stretched ion trap.

We were able to apply our simulation program immediately to an examination of single-ion trajectories in the stretched ion trap, and we have shown that the existence of a nonlinear resonance in the ion trap induces a change in ion secular frequencies as the ion moves away from the center of the ion trap [34]. (At the present time we are not aware of any other group that has the facility to calculate accurately ion trajectories in the commercial device.) Thus, when an ion is resonantly excited in the commercial device, it can go off resonance. Such behavior leads to both asymmetry in the absorption peak shape and an increase in the induction fluence, which, in part, explains the discrepancy in induction fluences discussed above. Another outcome of our simulation studies was that John Todd's group was encouraged to examine closely the temporal variation of components of their scan functions. They found that the duration of irradiation at a given tickle voltage amplitude was less than that assumed; it takes time for the full amplitude to be attained, thus the induction fluence had been overestimated in the experimental study, and agreement between experiment and simulation is much improved.

In a discussion of a single critical collision of an ion of $m / z 207$ with a helium atom, presented below it is shown that a significant increase in azimuthal kinetic energy as a result of the collision caused the ion to be lost from the ion trap. This observation has focused 
attention on the requirement for adequate cooling of ions prior to ion isolation to enhance the overall flexibility of this process; such enhancement has been confirmed experimentally [37].

\section{Overview of the System of Programs}

The system of programs employed in these simulation studies has been written in a combination of FORTRAN, BASIC, and 80386/80387 assembly language using Microsoft compilers and assemblers for use on 80386- and 80486-based microcomputers. One distinct advantage of using Microsoft compilers is the ease with which mixed-language programming can be implemented. In general, the calculation-intensive simulators have been coded in FORTRAN to take advantage of the computational speed of the executable code generated by FORTRAN optimizing compilers as well as the large number of readily available libraries of FORTRAN functions and subroutines for numerical analysis. Assembler functions and subroutines have been written to increase the speed of certain mathematical and data-transfer operations, and to gain access to system resources that are unavailable in FORTRAN. BASIC has been used to code those routines that are less calculation intensive and/or make extensive use of system resources.

The plotting package, used to display results, was written in BASIC but makes extensive use of assembly language subroutines to display graphical information on peripheral devices such as CRT displays, printers, and plotters.

Many of the useful insights that have been gained through the use of these simulation programs have resulted from performing a series of runs in which only a few parameters are varied. To facilitate this use of the simulators, a control system has been devised that allows a long series of runs to be carried out with no user intervention. Under this system, a data file is prepared by the user which specifies initial conditions for the first run and only those parameters that are to be changed in subsequent runs of a given series. A BASIC control program reads this data file, prepares an input data file for the appropriate simulator, and initiates a run. At the end of each run, the control program processes the files output by the simulator, prepares the next input data file, and initiates the next run. This process continues until the control program runs out of data, marking completion of the series. Simple batch programs are often employed to initiate several related series of simulations using control data files prepared by other programs.

Each individual run in a series consists of the following steps.

1. A generically named, run-time data file, which contains all the input parameters necessary to describe unambiguously a particular run, is prepared for the appropriate simulator by the control program. This run-time data file can be constructed in one of two ways dependent on a command-line option of the control program. In one mode of operation the control program builds each run-time data file such that each run represents some modification of the first run of the series. In the other mode, the control program uses a file of final conditions output by the simulator, with some modifications, as input for the next run. This latter mode is used to simulate, for example, ion isolation experiments in which each run in the series comprises one segment of a complex, but continuous, scan function operating on a single ion.

2. The appropriate simulator is invoked using the input data file prepared by the control program. During execution, the simulator writes binary data files for display of specified trajectory parameters as a function of time. Upon completion of a run, the simulator writes two ASCII files, one of which can be used, without modification, to continue the simulation where it left off and another that gives a readable description of trap operation during the run.

3. The control program invokes a batch file with a number of input parameters that carries out various operations on the generically named data files generated by the simulator. These operations can include the display of trajectory data; manipulation and /or processing of the data to obtain, for example, power spectra; and storage of data files using unique filenames.

4. Steps 1, 2, and 3 are repeated until the control program reaches the end of its input data file.

The FORTRAN simulators obtain their input data from a single ASCII file. The parameters that are specified in this data file, and, therefore, can be readily changed by the user, are:

1. Mass, charge, and initial phase-space coordinates of the ion

2. Mass, temperature, pressure, and polarizability volume of the inert collision gas

3. Initial and final stability coordinates, $\left(a_{z}, q_{z}\right.$ ) (Alternatively, these coordinates can be specified as DC and RF potentials.)

4. Amplitude and frequency of the $\mathrm{AC}$ auxiliary potential

5. Initial phases of the AC potentials

6. Electrode configuration, that is, percent hexapole and octupole in the multipole expansion of the potential inside the trap

7. Mode of application of the auxiliary potential, that is, monopolar, dipolar, or quadrupolar

8. Frequency and type of output

9. Various error control parameters appropriate to the particular solver routine in use 
Not all of the parameters itemized above are applicable to each simulator; however, for simplicity, all runtime data files have the same structure, with each simulator selecting only those parameters appropriate to its own use.

As in many computer applications, there exists here the tendency to generate an unmanageable amount of data in a very short period of time. When initially conceived, the simulators were used to examine, in detail, the trajectory of an ion over a relatively short period of time, spanning a few hundred RF cycles. Under these conditions, it was practical to output results (i.e., trajectory coordinates) at intervals corresponding to a small fraction of an RF period. As our applications became more sophisticated, with a single series of runs covering many segments of several milliseconds each, it became necessary to reduce the amount of output without sacrificing either accuracy or the ability to examine the fine details of an ion's motion. To resolve these apparently conflicting criteria, a compromise strategy was developed. One of the input parameters in the run-time data file is an output-control word whose individual bits are used to enable/disable specific output functions. Thus, over particular segments of a series in which ion behavior is both well-known and uninteresting, little or no output need be generated.

Segments where trajectory details are of interest can often be quite long. However, the most interesting portion of a segment is usually that which spans the final few periods of an ion's secular motion, for example. just before it strikes an electrode. When trajectory information covering a relatively long time period is required, points in an ion's trajectory are written to disk, typically once every RF cycle. Depending on input parameters, these points can be the result of a single calculation of the ion's phase-space coordinates, or the average of many calculations performed over that time-step. The end of a segment (i.e., an individual run) occurs either when the ion strikes an electrode or when a certain time limit has been reached. At this point, the simulator has the facility to recalculate, in greater detail, the ion's trajectory over the final few periods of the ion's secular motion prior to the end of the segment. The information required to restart the calculation at some earlier time in the segment is obtained from a circular queue of previous phase-space coordinates that is maintained for that purpose during the "coarse" phase of the calculation. Again, depending on input parameters, each of the "detailed-output" data points (usually 20-50 per RF cycle) can be the result of a single calculation, or the average of many.

Let us examine two trajectory simulations. The first calculation was carried out for an ion stored under the influence of an RF drive voltage of fixed amplitude and zero DC voltage, that is, at a fixed working point on the stability diagram. The second was carried out under the influence of a $D C$ voltage ramp while the $R F$ drive voltage amplitude was held constant, that is, as the working point was being moved vertically on the stability diagram.

The trajectory for an ion of $m / z 146$ confined within a quadrupole ion trap was calculated for a period of $200 \mu \mathrm{s}$ in time-steps of $9.091 \times 10^{-7} \mathrm{~s}$; the regular temporal variation of the ion kinetic energy over this period is shown in Figure 2a. There are 220 data points in this plot, that is, one per RF cycle; consequently, the rapid fluctuations in energy due to the $R F$ drive are not apparent. As discussed above, the trajectory was then recalculated over the final $40 \mu$ s (i.e., from $1.6 \times$ $10^{-4}$ to $2.0 \times 10^{-4} \mathrm{~s}$ ) in time-steps of $40 \mathrm{~ns}$, and the temporal variations of ion kinetic energy, radial position; and axial position relative to the center of the ion trap are shown in Figure $2 b-d$, respectively. Each of these plots is composed of $10^{3}$ data points. We see from Figure $2 \mathrm{~b}$ that the ion kinetic energy varied rapidly under the influence of the $R F$ drive voltage oscillating at a frequency of $1.1 \mathrm{mHz}$, and that kinetic energy maxima of approximately $0.16 \mathrm{eV}$ were attained compared to maxima of $0.055 \mathrm{eV}$ in Figure $2 \mathrm{a}$. The fundamental radial and axial secular frequencies are clearly evident in Figure $2 c$ and $d$, respectively, together with the superimposed RF drive frequency. Note that the radial secular frequency is approximately half that of the axial secular frequency, bearing in mind that, for radial motion, one cycle corresponds to the time interval between every second maximum [31].

The second trajectory calculation was made for an ion of $m / z 148$ as it was about to be ejected from the ion trap. The trajectory of the ion was stable initially but, as a DC ramp was applied to the ring electrode, the working point of the ion passed through the $\boldsymbol{\beta}_{r}=0$ boundary of the stability diagram. In Figure $3 a-c$ are shown the temporal variations of ion kinetic energy, radial and axial position, respectively, during a period of $40 \mu \mathrm{s}$ in time-steps of $40 \mathrm{~ns}$ while the DC voltage was ramped. The same amount of detail is shown in Figure $3 a-c$ as is shown in Figure $2 b-d$. Both ion kinetic energy and radial position are seen to increase during this period; ion kinetic energy is approximately $15 \mathrm{eV}$ while a radial excursion of $3.5 \mathrm{~mm}$ was attained. During this period, axial excursions were restricted to less than $1 \mathrm{~mm}$ from the center of the ion trap.

\section{Implementation of Collisions}

Collisions between an ion and atoms of an inert cooling gas are simulated by using the Langevin Theory. In Appendix $A$ the details of this theory are presented and the appropriate collision cross-section is derived (eq A5). Conveniently, the probability of a collision per unit time (eq A7) is independent of the relative velocity of the collision partners and need be calculated only once at the beginning of a run. 

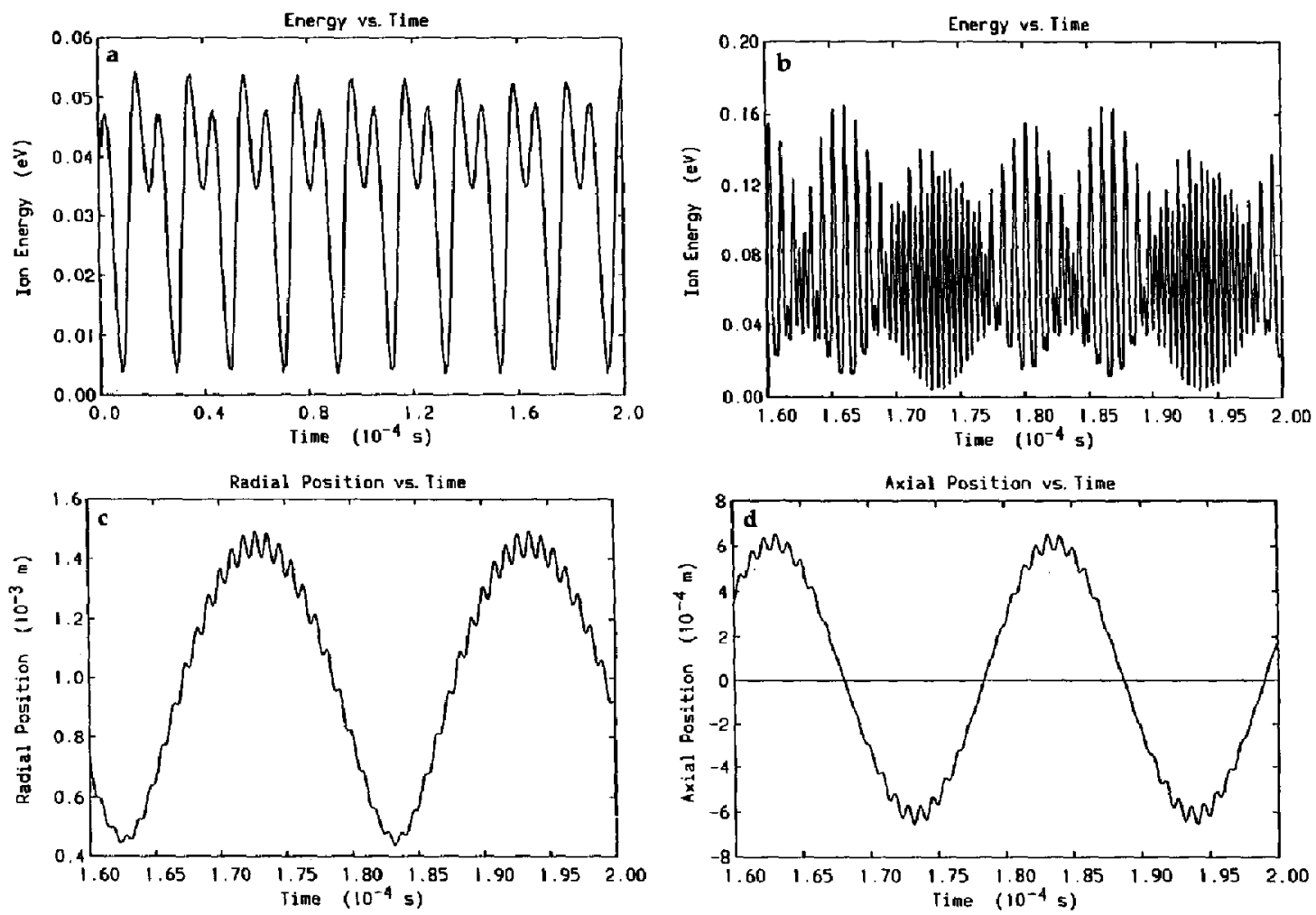

Figure 2. Trajectory simulation of $m / z 146$ at $\left(a_{z}, q_{z}\right)=(0,0.1245)$. (a) Kinetic energy variation over the time period $0-200 \mu \mathrm{s}$ in time steps of $9.091 \times 10^{-7} \mathrm{~s}$; (b) kinetic energy variation over the time period 160-200 $\mu \mathrm{s}$ in time steps of $40 \mathrm{~ns}$; (c) radial excursions over the time period $160-200 \mu \mathrm{s}$; (d) axial excursions over the time period 160-200 $\mu \mathrm{s}$.

Random collisions are implemented in the following way.

1. At each time-step in the calculation a random number between zero and one is generated. If this number is less than or equal to the probability of a collision occurring over the period of one time-step, then a collision is deemed to have occurred.

2. In some previous work the magnitude of the velocity of the collision-gas was assumed to be simply the average Maxwell-Boltzmann velocity at the temperature of the gas. However, in recent ion-isolation simulations, the random nature of collisions has proven to be an important factor in determining the behavior of ions near the boundaries of stability in $(a, q)$ space. Consequently, the collision algorithm was refined such that the magnitude of the velocity of the collision gas is chosen randomly from a Maxwell-Boltzmann distribution of velocities.

3. The collision-gas atom is given a random direction in the laboratory reference frame.

4. The velocity of the center of mass is determined.

5. The magnitude of the velocity of the ion in the center of mass reference frame is calculated. (For elastic collisions, in the center of mass frame, only the directions of the velocities of the collision partners are changed by the collision. The magnitudes of the velocities remain unchanged.)

6. The scattered ion is given a random direction in the center of mass frame. This is equivalent to choosing a random value for the impact parameter that varies between zero and the maximum value for which a capture will occur.

7. The scattered ion's new velocity is transformed to the laboratory reference frame.

The role of collisions in an ion trap between confined ions and the buffer gas is complex in that collisions fulfill several functions which determine the overall behavior of confined ions. The most dramatic function is that of dissipation of ion momentum, whereby ions become collisionally focused near the center of the ion trap. Ions of lower $m / z$ are focused to the center more rapidly than are ions of higher $m / z$, which resembles the concentric layers of an onion. It is 

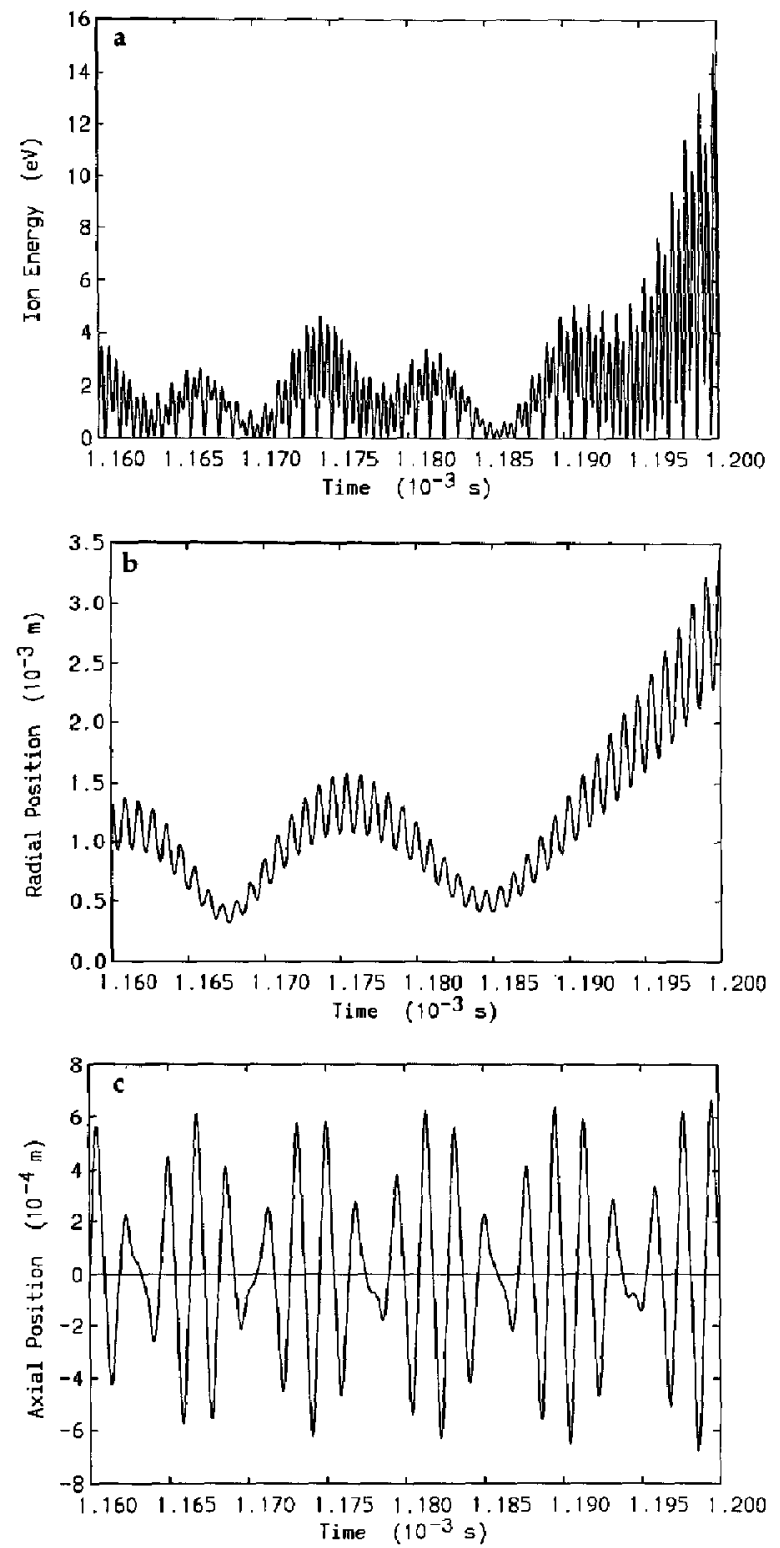

Figure 3. Trajectory simulation of $m / z$ 148, with $q_{z}=0.7692$, during application of, and immediately following, the DC ramp. $a_{z}$ is increased from zero to $0.1494 \times \frac{146}{148}$. (The final working point lies only just outside the stability diagram.) (a) Kinetic energy variation over the time period $1160-1200 \mu \mathrm{s}$ in time steps of $40 \mathrm{~ns}$; (b) radial excursions over the time period 1160-1200 $\mu \mathrm{s}$; (c) axial excursions over the time period 1160-1200 $\mu \mathrm{s}$.

necessary, in special circumstances, to take this model into account when carrying out mass-selective ion ejection in order of increasing $m / z$ order, as ions of lower $m / z$ that are to be excited axially for ejection must pass through the layers of ions of higher $\mathrm{m} / z$; in so doing, they experience the space charge of the higher $m / z$ ions, which can perturb ion trajectories.

Collisions also serve to bring about conversion of ion kinetic energy to internal energy, which can result in ion dissociation; this vibrational excitation mechanism is moderated by other competing collisions, which lead to deactivation of vibration energy. Such inelastic collisions bring about changes in the magnitudes of the radial and axial components of ion motion, and when the working point of an ion lies in the vicinity of a stability boundary, these changes in the components of motion can induce trajectory instability, resulting in loss of the ion by collision with an electrode.

Two examples of collisions are illustrated here. First is the case of multiple collisions by which ion momentum is lost in a series of collisions so that the ion motion is cooled and the ion is confined near the center of the ion trap. Second we examine a single collision in detail and find that, under appropriate experimental conditions, relatively small changes in the components of ion motion can lead to loss of the ion.

The trajectory was calculated for an ion of $m / z 207$ with a working point located on the $q_{z}$ axis at $q_{z}=0.2$ (see below). The ion had relatively large secular amplitudes, both radial and axial, and consequently was highly energetic. The trajectory of the ion was calculated initially for $50 \mu \mathrm{s}$ in a collision-free system to verify trajectory stability; such a procedure is always necessary unless one has verified previously that the initial conditions of ion position, velocity, drive voltage amplitude and phase result in a stable trajectory for the subject ion. During the first microsecond of ion motion unimpeded by collisions, the ion exhibited a maximum kinetic energy of $33 \mathrm{eV}$ and maximum radial and axial excursions of 7.7 and $6.2 \mathrm{~mm}$, respectively. Thus, the ion was energy rich and its trajectory filled the major portion of the ion trap.

In the simulation, helium gas was then added at a pressure of $1.5 \times 10^{-2}$ torr; this relatively high pressure of helium was chosen to permit foreshortening of the computational time. The motion of the ion was not unduly restricted at this pressure as, on average, there were 88 time-steps between successive collisions, that is, the probability of a collision was 0.012 for each time-step of $4.545 \times 10^{-8} \mathrm{~s}$. The trajectory was calculated for $2000 \mu s$ at this helium pressure. The instantaneous values of ion kinetic energy before and after each of the 510 collisions are shown in Figure 4; the inset shows the kinetic energy averaged over each RF cycle during the same time period. Maximum damping of ion kinetic energy and, though not shown here, radial and axial excursions were seen to have been achieved after approximately $1000 \mu \mathrm{s}$; thereafter, the ion remained essentially quiescent.

Let us now examine a single, and critical, collision between an ion of $m / z 207$ and a helium atom. The working point of the ion lies in the vicinity of the $\beta_{z}=0$ boundary of the stability diagram. The radial, 


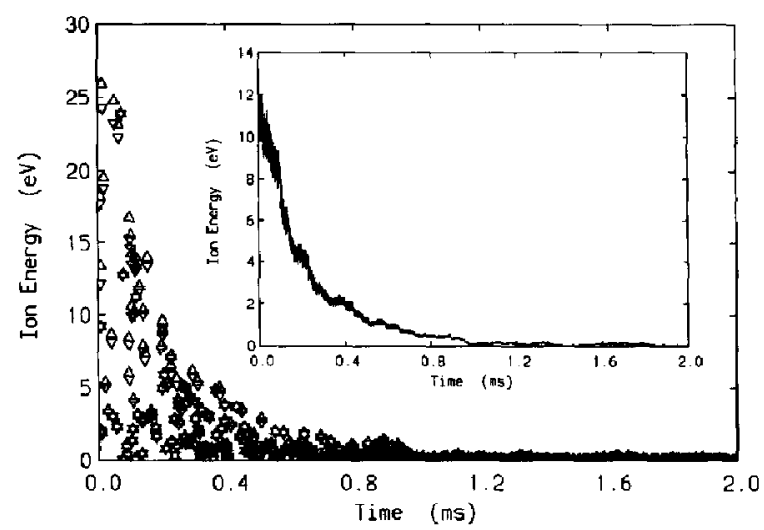

Figure 4. Trajectory simulations of $m / z 207$, with collisions corresponding to a pressure of helium of $1.5 \times 10^{-2}$ torr, at the working point $\left(a_{z}, q_{z}\right)=(0,0.2)$, over a time period of $2000 \mu \mathrm{s}$. Upright and inverted triangles correspond, respectively, to instantaneous ion kinetic energies immediately prior to and following a collision. Inset shows ion kinetic energy averaged over each RF cycle.

azimuthal (orbital in a radial plane), and axial energies are $0.00103 \mathrm{eV}, 0.00198 \mathrm{eV}$, and $0.165 \mathrm{eV}$, respectively, prior to the collision; after the collision, the same energies are $0.000675 \mathrm{eV}, 0.00695 \mathrm{eV}$, and $0.131 \mathrm{eV}$, respectively. The collision brought about losses of radial and axial energies and an increase of $251 \%$ in the relatively low arimuthal energy; there was a net loss of kinetic energy.

This collision occurred at $1.91324 \mathrm{~ms}$ of a simulation. The net result of these changes in component kinetic energies was that the trajectory of the ion eventually exceeded the physical dimensions of the trap even though the absolute changes in component kinetic energies were minor. In the accompanying figures, we have attempted to show the behavior of the ion prior to and subsequent to the collision and the immediate changes in component kinetic energies wrought by the collision. Furthermore, in some figures, we have indicated not only the behavior of the ion subsequent to the collision, but the behavior of the ion had the collision not occurred so as to illustrate the marked effect of the field on the ion subsequent to the minor changes in ion motion wrought by the collision.

In Figure $5 \mathrm{a}$ is shown the temporal variation of the ion kinetic energy over a simulation period of $100 \mu \mathrm{s}$, from 1.86-1.96 ms; the collisional event is marked at $1.91324 \mathrm{~ms}$ with upright and inverted triangles which represent kinetic energy prior to and subsequent to the collision, respectively. The triangles are overlapped due to the energy scale used here.

While the change in kinetic energy due to the collision alone was minute compared with the ordinate scale of Figure 5a, the subsequent action of the field upon the ion had a marked effect in that ion kinetic energy maxima were increased subsequently threefold. In Figure $5 b$ the time and energy scales have been diminished to show the loss in kinetic energy as a
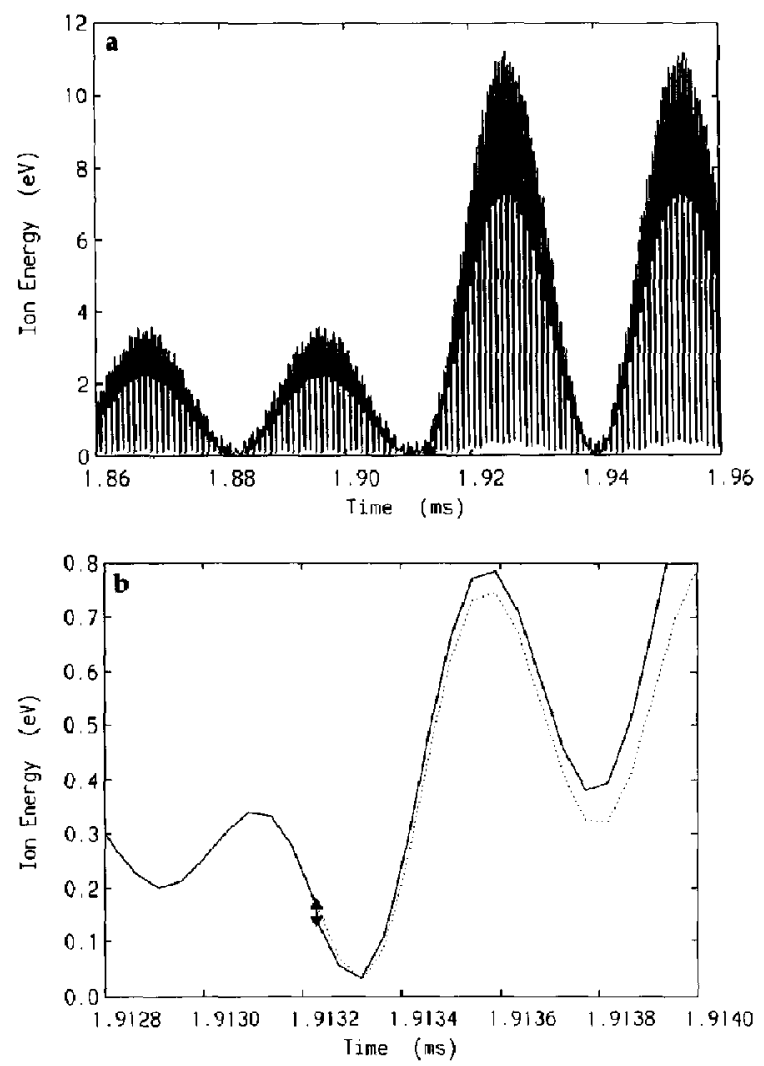

Figure 5. Temporal variation of ion kinetic energy of an ion of $m / z 207$ prior to and after a collision. (a) Over a simulation period of $100 \mu \mathrm{s}$, from 1.86-1.96 ms; (b) over a simulation period of $1.2 \mu \mathrm{s}$, from $1.9128-1.9140 \mathrm{~ms}$ (note that a collision occurred at $1.91324 \mathrm{~ms}$ ). The upright and inverted triangles represent kinetic energy prior to and subsequent to the collision, respectively. The dotted line in $\mathbf{b}$ shows the temporal variation of ion kinetic energy had the collision not occurred.

result of the collision. The solid line shows ion kinetic energy prior to, at, and following the collision, while the dotted line shows ion kinetic energy had the collision not occurred.

In Figure $6 a$ and $b$ are shown the radial and azimuthal kinetic energies, respectively, over a simulation period of $10 \mu \mathrm{s}$. The loss in radial kinetic energy is minute again on the scale of Figure $6 \mathrm{a}$ so that the triangles are overlapped. The dotted line shows that the collision had little effect upon subsequent radial kinetic energy. The gain in azimuthal kinetic energy as a result of the collision is seen clearly in Figure $6 \mathrm{~b}$ and, in comparison with the dotted line, is seen to persist following the collision. An expanded view of the changes in azimuthal energies prior to, at, and after the collision are shown in Figure 6c.

The temporal variation of ion axial kinetic energy shown in Figure 7a covers a simulation period of 100 $\mu \mathrm{s}$. The time of collision is shown by the overlapping triangles. The temporal variation shown here is quite 

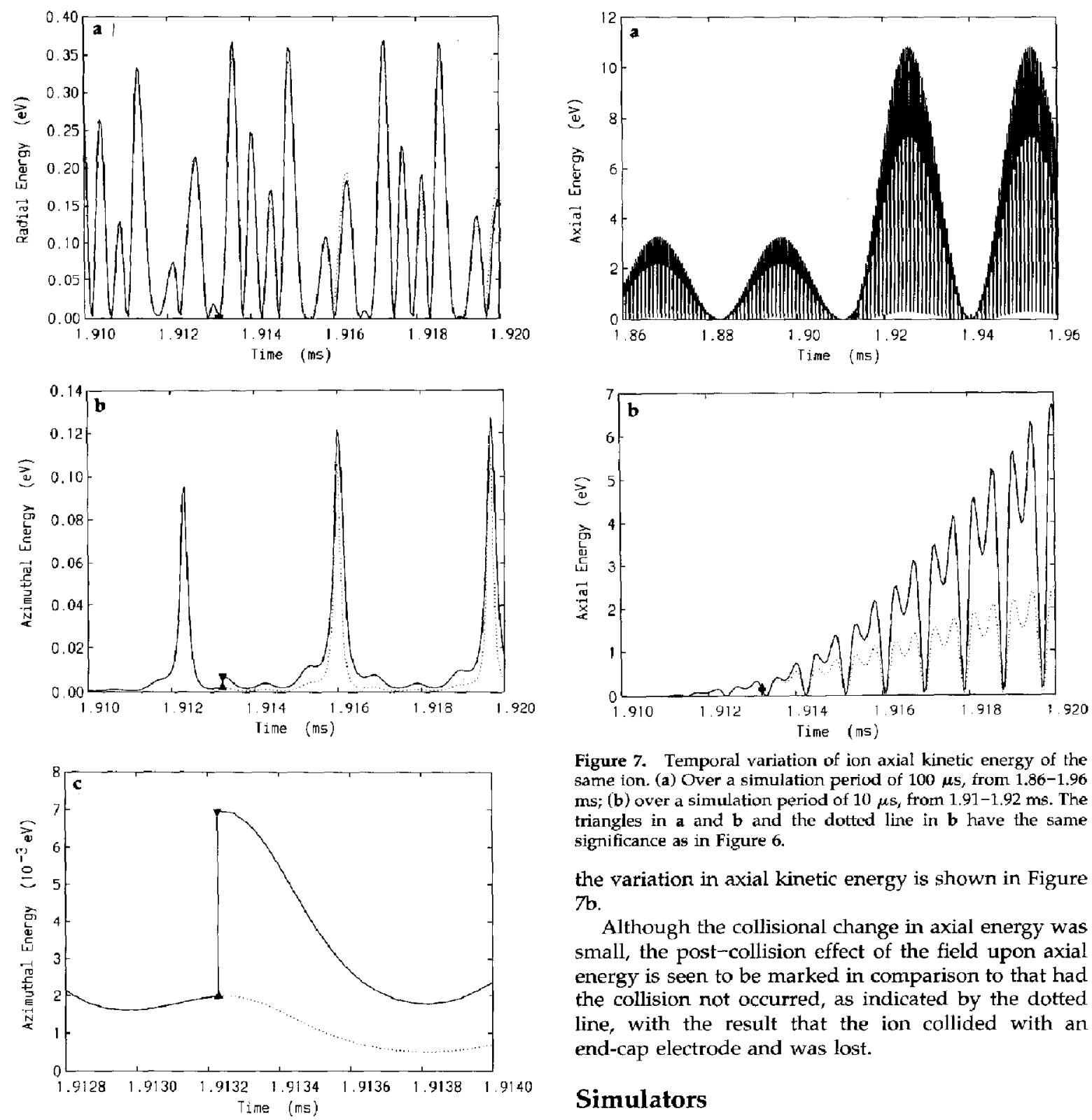

Figure 6. Temporal variation of radial and azimuthal components of the kinetic energy of the same ion as in Figure 5. (a) Radial kinetic energy over a simulation period of $10 \mu \mathrm{s}$, from 1.91-1.92 ms; (b) azimuthal kinetic energy over the simulation period $1.9128-1.9140 \mathrm{~ms}$, that is, $1.2 \mu \mathrm{s}$. In $\mathbf{a}$ and $\mathbf{b}$ the triangles depict collisional kinetic energy values while the dotted lines show the behavior of the energy components had the collision not occurred.

similar to that of ion kinetic energy shown in Figure 5a as the bulk of the ion kinetic energy resides in axial motion. However, the variations in axial kinetic energy minima and maxima are more smooth than those for total ion kinetic energy. A magnified presentation of

Figure 7. Temporal variation of ion axial kinetic energy of the same fon. (a) Over a simulation period of $100 \mu \mathrm{s}$, from 1.86-1.96 $\mathrm{ms}$; (b) over a simulation period of $10 \mu \mathrm{s}$, from 1.91-1.92 $\mathrm{ms}$. The triangles in $a$ and $b$ and the dotted line in $\mathbf{b}$ have the same significance as in Figure 6.

the variation in axial kinetic energy is shown in Figure $7 \mathrm{~b}$.

Although the collisional change in axial energy was small, the post-collision effect of the field upon axial energy is seen to be marked in comparison to that had the collision not occurred, as indicated by the dotted line, with the result that the ion collided with an end-cap electrode and was lost.

\section{Simulators}

\section{Simulator MA}

Simulation program MA uses the analytic solution of the Mathieu equation to calculate ion trajectories. By definition, pure quadrupolar fields are assumed and provision for the inclusion of an auxiliary potential exists* [38]. MA is used primarily as a check against

* Recent theoretical work has resulted in two modified versions of MA which use first-order perturbation theory to produce approximate analytic expressions that can be used to calculate an ion's trajectory when an auxiliary voltage is applied in quadrupolar mode. These solutions follow the same general form as the Mathieu solution; however, it is beyond the scope of this article to report the results here. 
the operation of the other simulators operating under these restricted conditions; it offers the advantage of calculating the details of an ion's trajectory directly at any given point in time with great accuracy without the necessity of taking a large number of program steps to reach it. The theory for this simulator is presented below.

In an undistorted, purely quadrupolar, ion trap the electric potential is given by eq B22 in Appendix B. The differential equation of motion for a singly charged, positive ion subject to this potential is readily obtained from

$$
\frac{d^{2} \vec{r}}{d t^{2}}=-\frac{e}{m} \nabla \Phi
$$

where $m$ is the mass of the ion and $e$ is the electronic charge.

In this discussion it will be assumed that both end-cap electrodes are grounded and that an electric potential of

$$
\Phi_{0}^{R}=u+V \cos (\Omega t+\gamma)
$$

is applied to the ring electrode, where $U$ and $V$ are, respectively, the amplitudes of the direct and altemating components of $\Phi_{0}^{R}$ and $\Omega$ is the angular frequency of the alternating component with an initial phase angle of $\gamma$. Then, eq 1 can be written as

$$
\frac{d^{2} u}{d t^{2}}=\frac{2 \alpha e}{m\left(\rho_{0}^{2}+2 z_{0}^{2}\right)}[u+V \cos (\Omega t+\gamma)] u
$$

where $\alpha=1$ for $u=\rho$ and $\alpha=-2$ for $u=z$. With $\gamma$ set to zero for simplicity, eq 3 can be cast in the canonical form of the Mathieu equation:

$$
\frac{d^{2} u_{0}}{d \xi^{2}}+\left[a_{u}-2 q_{u} \cos (2 \xi)\right] u_{0}=0
$$

where $\xi=\frac{\Omega t}{2}$, and the stability parameters $a_{u}$ and $q_{u}$ are given by

$$
\begin{aligned}
& a_{\rho}=\frac{8 e U}{m\left(\rho_{0}^{2}+2 z_{0}^{2}\right) \Omega^{2}} \\
& a_{z}=\frac{-16 e U}{m\left(\rho_{0}^{2}+2 z_{0}^{2}\right) \Omega^{2}} \\
& q_{\rho}=\frac{-4 e V}{m\left(\rho_{0}^{2}+2 z_{0}^{2}\right) \Omega^{2}}
\end{aligned}
$$

and

$$
q_{z}=\frac{8 e V}{m\left(\rho_{0}^{2}+2 z_{0}^{2}\right) \Omega^{2}}
$$

The stable solutions of eq 4 are known to have the general form [39]

$$
u_{0}(\xi)=A_{u} u_{0}^{C}(\xi)+B_{u} u_{0}^{S}(\xi)
$$

where $A_{1 \alpha}$ and $B_{u}$ are arbitrary constants and

$$
u_{0}^{C}(\xi)=\sum_{n=-\infty}^{+\infty} C_{2 n, u} \cos \left(2 n+\beta_{u}\right) \xi
$$

and

$$
u_{0}^{s}(\xi)=\sum_{n=-\infty}^{+\infty} C_{2 n, u} \sin \left(2 n+\beta_{u}\right) \xi
$$

with $\beta_{u}$ determining the spectrum of frequencies corresponding to the parameter pair $\left(a_{u}, q_{u}\right)$. The $C_{2 n, u}$ coefficients give the amplitudes of the allowed modes in the spectral analysis of $u_{0}$.

$\beta_{u}$ can be calculated from $a_{u}$ and $\eta_{u}$ using the continued fraction expression

$$
\begin{aligned}
\beta_{u}^{2}=a_{u}+\frac{q_{u}^{2}}{\left(\beta_{u}+2\right)^{2}-a_{u}-\frac{q_{u}^{2}}{\left(\beta_{u}+4\right)^{2}-a_{u}-\frac{q_{u}^{2}}{\left(\beta_{u}+6\right)^{2}-a_{u}-\ldots}}} \\
+\frac{q_{u}^{2}}{\left(\beta_{u}-2\right)^{2}-a_{u}--\frac{q_{u}^{2}}{\left(\beta_{u}-4\right)^{2}-a_{u}-\frac{q_{u}^{2}}{\left(\beta_{u}-6\right)^{2}-a_{u}-\ldots}-}}
\end{aligned}
$$

Knowing $\beta_{u}$, the $C_{2 r, u}$ coefficients can be calculated, assuming $C_{0, u}=1$, from the recursion formulae

$$
C_{2 n, u}=\frac{-C_{2 n-2, u}}{q_{u}}-\frac{q_{u}^{2}}{\left(2 n+\beta_{u}\right)^{2}-a_{u}-\frac{q_{u}^{2}}{\left(2 n+2+\beta_{u}\right)^{2}-a_{u}-\frac{q_{u}^{2}}{\left(2 n+4+\beta_{u}\right)^{2}-a_{u}-\ldots}}}
$$


and

$$
C_{2 n, u}=\frac{-C_{2 n+2, u}}{q_{u}} \frac{q_{u}^{2}}{\left(2 n+\beta_{u}\right)^{2}-a_{u}-\frac{2_{u}^{2}}{\left(2 n-2+\beta_{u}\right)^{2}-a_{u}-\frac{q_{u}^{2}}{\left(2 n-4+\beta_{u}\right)^{2}-a_{u}-\ldots}}}
$$

$\beta_{u}$ and the $C_{2 n_{u} u}$ are calculated to the limits of accuracy imposed by double precision arithmetic (IEEE 8-byte reals) using the above formulae. $A_{u}$ and $B_{u}$ are calculated from the initial conditions given by $u$ and $\dot{u}$. In all cases, sufficient terms are calculated in the summations that the inclusion of additional terms would not change the result represented as an IEEE 8-byte real. Execution time is a function of the number of points at which position and velocity are calculated.

\section{Simulator $S P Q R$}

Simulator SPQR is used to calculate single-ion trajectories for configurations where the RF drive potential, $\Phi_{0}^{R}$, is applied to the ring electrode and the auxiliary (tickle) potential, $\Phi_{0}^{E}$, is applied, in phase, to both end-cap electrodes (quadrupolar mode). Both of these potentials are assumed to be of the form given by eq 2 with the DC component of $\Phi_{0}^{E}$ set to zero.

Following eq 1 and using the expression for the potential given by eq B8, the equations of motion for a singly charged positive ion, expressed in Cartesian coordinates, are

$$
\frac{d^{2} x}{d t^{2}}+\frac{2 e A}{m}\left[1+3 A_{3} z+2 A_{4}\left(x^{2}+y^{2}-4 z^{2}\right)\right] x=0
$$

$$
\frac{d^{2} y}{d t^{2}}+\frac{2 e A}{m}\left[1+3 A_{3} z+2 A_{4}\left(x^{2}+y^{2}-4 z^{2}\right)\right] y=0
$$

and

$$
\begin{gathered}
\frac{d^{2} z}{d t^{2}}+\frac{2 e A}{m}\left[-2 z+\frac{3}{2} A_{3}\left(x^{2}+y^{2}-2 z^{2}\right)\right. \\
\left.-8 A_{4}\left(x^{2}+y^{2}-\frac{2}{3} z^{2}\right) z\right]=0
\end{gathered}
$$

where $A, A_{3}$, and $A_{4}$ are obtained from eqs B19, B31, and $B 32$, respectively.

Ion trajectories are calculated by numerically integrating eqs $15-17$ for which the three second-order, differential equations are cast in the form of six firstorder, differential equations. For example, eq 15 can be expressed as

$$
\begin{aligned}
& \frac{d x}{d t}=\dot{x} \& \\
& \frac{d \dot{x}}{d t}=-\frac{2 e A}{m}\left[1+3 A_{3} z+2 A_{4}\left(x^{2}+y^{2}-4 z^{2}\right)\right] x
\end{aligned}
$$

The resulting set of six first-order, differential equations are solved using the Bulirsch-Stoer method for numerical integration with adaptive step-size and error control [40]. By using this technique it is possible to take very large time-steps, of the order of one cycle of the RF drive potential, between calls to the solver routine without sacrificing accuracy. Smaller timesteps, which are often desirable for reasons other than accuracy of calculated trajectories, increase execution time proportionately. However, because the solver routine optimizes step size automatically, increasing the period between calls beyond one RF cycle does not reduce execution time significantly. In general, large time-steps can be used during relatively uninteresting segments of a simulation, for example, to relocate an ion's working point on the stability diagram. Smaller time steps, ranging from onc-twenticth to one fifticth of an RF cycle, are used for segments where details of an ion's RF motion are important.

Execution time for a simulation segment is a function of the total time period covered by the calculation, the frequency with which results are written to disk, the accuracy to which positions and velocities are determined, and the size of the time increment between calls to the solver routine.

\section{Simulator FIM}

This simulator incorporates all the features of SPQR with the added capability of modeling monopolar and dipolar modes of applying an auxiliary potential. The disadvantages of using FIM are greater complexity, increased execution time, and the relatively large number of electrode-shape-specific support files required at run-time.

Regardless of the percentage of hexapole and octupole field-components which are present inside an ion trap, an analytic, differentiable expression can be written down which describes exactly the potential for quadrupolar-mode auxiliary-potential application. This 
condition does not hold for the monopolar and dipolar modes. As a consequence, no closed expression can be obtained for the force which an ion experiences at a general point inside the trap under either of these modes of operation. By using a technique which is described below, electric field components for configurations of interest are precalculated at evenly spaced grid points inside the trap and stored in library files. At run-time, the field-component matrices appropriate to the specified configuration are selected from the library and the electric field-component values for points on an ion's trajectory are obtained by performing two-dimensional, polynomial interpolations.

The FIM simulator calculates new values for an ion's position and velocity, $(\vec{r}, \vec{\nu})$, from coordinates calculated on the previous step, $\left(\vec{r}_{0}, \vec{v}_{0}\right)$, by using the elementary equations

$$
\vec{r}=\vec{r}_{0}+\vec{\nu}_{0}(\delta t)+\frac{1}{2} \vec{a}(\delta t)^{2} \& \vec{\nu}=\vec{\nu}_{0}+\vec{a}(\delta t)
$$

where $\delta t$ is the time increment between calculations of phase-space coordinates, and the acceleration for a singly charged positive ion, $\vec{a}=\frac{e}{m} \vec{E}(t)$, depends on time through the electric field, $\vec{E}(t)$.

When the RF drive potential can be described by the multipole expansion given by eq B21, the electric field components due to the RF drive potential applied to the ring clectrode, $\Phi_{0}^{R}(t)$, can be calculated directly from the gradient of the potential as

$$
\begin{aligned}
E_{x}(t)= & \frac{-2 \Phi_{0}^{R^{2}}(t)}{(\lambda+\mu) \rho_{0}^{2}} \\
& \times\left[1+3 A_{3} z+2 A_{4}\left(x^{2}+y^{2}-4 z^{2}\right)\right] x \\
L_{y}(t)= & \frac{-2 \Phi_{0}^{R}(t)}{(\lambda+\mu) \rho_{0}^{2}} \\
& \times\left[1+3 A_{3} z+2 A_{4}\left(x^{2}+y^{2}-4 z^{2}\right)\right] y
\end{aligned}
$$

and

$$
\begin{aligned}
E_{z}(t)= & \frac{-2 \Phi_{0}^{R}(t)}{(\lambda+\mu) \rho_{0}^{2}}\left[-2 z-\frac{3}{2} A_{3}\left(x^{2}+y^{2}-2 z^{2}\right)\right. \\
& \left.+8 A_{4}\left(x^{2}+y^{2}-4 z^{2}\right) z\right]
\end{aligned}
$$

where $\Phi_{0}^{R}(t)$ is given by eq 2 . The electric field components due to the auxiliary potential, $\Phi_{n}^{E}(t)$, are determined from matrices of preassigned values using twodimensional interpolations, and combined with the RF drive field-components according to the principle of superposition. If the electrode geometries are such that the potential field of the RF drive cannot be written as a simple multipole expansion, then the electric field components due to the RF drive must be determined by the same technique as that used to obtain the auxiliary field.

The time required by the FIM simulator to calculate an ion's trajectory over a fixed time segment is primarily a function of the size of the time increment, $\delta t$, between calls to the solver routine, the order of the interpolation and, to a lesser extent, the frequency at which results are written to disk. Values for $\delta t$ are typically chosen between 0.001 and 0.005 cycles of the RF drive potential. Because errors tend to be cumulative with this method, relatively small increments are required if accuracy is to be maintained over a relatively long simulation period. Also, relatively smaller values of $\delta t$ are required for highly energetic ions, that is, ions with high velocities.

Construction of the electric field matrices. Because all traps modeled by these simulations have axial symmetry, nonzero electric fields exist only in the radial and axial directions. Therefore, only a two-dimensional calculation is required. The origin of a cylindrical coordinate system is assumed to lie at the geometric center of a purely quadrupolar trap. When field components are asymmetric about the $z=0$ plane, only the first quadrant of the $p-z$ plane need be considered; however, this condition does not hold for traps with nonzero hexapole field components and, in this case, it is necessary to extend the calculation over both positive and negative values of $z$. In gencral, calculations of ficld components are carried out between radial limits of $\rho=0$ and $\rho=\rho_{\max }$ and between axial limits of $z=$ $\pm z_{\max }$

The electric potential can be calculated numerically by using the relaxation method [41] at specific grid puints in any region of space where the potentials on the boundaries of that region are known. This technique has been applied to the calculation of the potential at grid points inside the ion trap for specific electrode configurations and modes of operation. The resulting matrix of electric potentials is differentiated along grid lines of constant $\rho$ and $z$ to obtain grid-point values of the axial and radial electric field components, respectively.

This technique, although very simple in principle, is complicated considerably by the nonrectilinear shapes of the electrodes. One option is to choose a relatively small grid-point spacing and approximate the electrode surfaces in a step-wise manner by defining each surface with a series of grid-points which approach, most closely, its true position. However, because the behavior of ions near the surface of the end-cap electrodes is very important in determining accurately exit times during both mass-instability scans and resonance excitation, a more rigorous method was adopted. In this work, the positions of the electrode surfaces have been represented exactly. Consequently, there is considerable computational overhead in keeping track of, and providing special treatment for, those grid points which lie adjacent to electrode surfaces. This approach 
does have the advantage of allowing a relatively large grid-point spacing without sacrificing accuracy near boundaries.

Calculation of the electric potential by the relaxation method. Before the field-component matrices can be obtained the potential inside the trap must be known. A program designated $\mathrm{PQHO}$ is used to calculate the potential at grid points inside traps which exhibit a mixture of quadrupolar, hexapolar, and octupolar components. The first step in this process is to define the electrode surfaces and assign potentials to them. The potential on each electrode is assigned a value of either 0 or 1 . Each mode of operation can be designated by a set of three numbers which correspond to the potentials assigned to the upper end-cap, ring, and lower end-cap electrodes respectively. By using this scheme, the quadrupolar mode can be designated by $(1,0,1)$, the dipolar mode by $(1,0,-1)$, and the monopolar modes by $(1,0,0)$ and $(0,0,1)$. It is noteworthy that the two monopolar modes are equivalent only when the trap is symmetric about the $z=0$ plane; such is the case only when the hexapole fieldcomponent fraction is zero. On the other hand, $(1,0,1)$ and $(0,1,0)$ configurations correspond to identical quadrupolar modes which differ from each other only by a phase angle of $180^{\circ}$. In practice it makes no difference whether a monopolar or dipolar mode of operation is modeled because the quadrupolar configuration is known analytically (see eq 11), and the unknown mode can be readily obtained from the other two by applying the principle of superposition, for example, $(1,0,-1)=(1,0,1)-2(0,0,1)$. For those cases where the quadrupolar mode can be calculated analytically from eq $B 8$, the relaxation method need only be applied to calculate one other mode.

Another consideration which results in a trade-off between execution speed and accuracy is the need to impose an artificial boundary condition where the calculation is truncated at $\rho_{\max }$. This is done by assuming that the potential varies linearly along the line $\rho=\rho_{\max }$ between the ring and end-cap electrodes. It then becomes necessary to choose $\rho_{\max }$ sufficiently large that fields in the ion-containment region of the trap are not distorted by this approximation. In practice, we have found that with $\rho_{\max }=6 \rho_{0}$ the potential in the ioncontainment region is undistorted within the limits of accuracy imposed by double precision arithmetic.

Another boundary of the calculation at which the potential is unknown is along the line defined by $\rho=0$. However, because of the symmetry of the trap about the $z$-axis, the potential along the line of grid points immediately to the left of the $z$-axis is identical to the potential along the line of grid points immediately to the right. Although this symmetry provides an adequate boundary condition for the calculation, convergence would proceed more rapidly if fixed values were known a priori.
Parameters input to program $\mathrm{PQHO}$ include the following:

1. The equatorial radius of the trap $p_{0}$

2. The hexapole and octupole field-component fractions $f_{3}$ and $f_{4}$ defined by eqs B29 and B30

3. The grid-point spacing (The $0.025 \rho_{0}$ has proven to be sufficiently small.)

4. The limiting value for the radial coordinate $\rho_{\max }$ (An appropriate value for $z_{\max }$ is calculated from $\rho_{\max }$ and the configuration geometry.)

5. The over-relaxation factor to be used in the calculation (A value of about 1.3 has been found to the fastest convergence.)

6. Electrode coordinates at intersections with grid lines when they cannot be calculated analytically

Steps in calculation of the electric potential (how $\mathrm{PQHO}$ works) include the following:

1. Coordinate values are assigned to grid points inside the trap.

2. The indices of grid points inside the trap which are closest to the electrode surfaces are determined.

3. The coordinates of electrode surfaces along grid lines are determined.

4. The results of $1-3$ are written to binary data files for use by subsequent programs.

5. An ASCII file of parameters required by subsequent programs to interpret the binary files written in 4 is written to disk.

6. Binary data files of $(\rho, z)$ pairs for plotting electrode surfaces are written to disk.

7. Boundary conditions for the potential are established at $\rho_{\max }$.

8. Approximate initial values for the potential at grid points are calculated by the method of collocation [42].

9. The relaxation method is used to calculate, iteratively, the potential at grid points until results are consistent within specified limits, typically 14 or 15 digits.

Note: Because the two-dimensional array of potentials is, in general, too large to be stored in memory, results are stored in a binary disk file. Only that portion of the array required to calculate potentials along a grid line of constant $\rho$ is kept in memory at any one time.

Field-component matrices. To avoid boundary truncation errors, the potential matrices calculated by $\mathrm{PQHO}$ extend far beyond the ion-containment region of the trap. Because there is no point in calculating clcctric fields in locations not visited by ions, the size of the potential matrix output by $\mathrm{PQHO}$, as well as the accompanying support files, are reduced in size such that $\rho_{\max }=1.5 \rho_{0}$, with a similar reduction in the value 
of $z_{\max }$. The electric field-component matrices are then constructed by differentiating the potential matrix along grid lines of constant $\rho$ and $z$ to obtain grid-point values of the axial and radial electric field components, respectively. Matrix construction is accomplished by two separate programs.

The first of these is a BASIC program which extracts segments of records from the potential-matrix file that lie inside the trap. Dependent upon command-line parameters, record segments of either constant $\rho$ or constant $z$ can be obtained. This program is called successively by a second program, written in BASIC with FORTRAN subroutines, which calculates the electric field and constructs matrices of the electric field components. This second program calculates field components by performing a tenth-order polynomial fit to the potential at 11 consecutive grid points, and the electric field component at the middle of the segment is calculated from the analytic derivative of the polynomial. The electric field components $\left(E_{p}\right.$ and $\left.E_{z}\right)$ at each grid point inside the trap and at each intersection of a grid line with an electrode surface are calculated by this method. Where fewer than 11 grid points exist along a grid line between two electrode surfaces, the order of the polynomial is reduced accordingly.

The field-component matrices prepared by this program are read directly by the simulator at run-time. All of the programs in this discussion, including the simulator, require access to the support files written by $\mathrm{PQHO}$ to interpret correctly the matrix files.

\section{Conclusions}

From an initial simulation program (MA) which calculated the collision-free and irradiation-free trajectories of a single ion trapped in three-dimensional quadrupolar fields, we have developed a system of programs for the calculation of trajectories under a variety of conditions. With the SPQR program, quadrupolar excitation could be simulated, while with the FIM program the mode of excitation could be varied to include monopolar and dipolar excitation.

SPQR and FIM were then modified to include collisions with neutral species of known polarizability and with a Maxwell-Boltzmann distribution of velocities. Recently, all of the features have been incorporated into a single program, ISIS, which has the facility to calculate single-ion trajectories in traps of known geometry, for example, one whose electrodes have been shaped to include specified percentages of hexapole and octupole components in the electric field inside the trap. Furthermore, ISIS has the capability to model ion-isolation experiments where the amplitude of the $D C$ and RF potentials are ramped as a function of time. In comparisons with experimental investigations of resonance excitation and $\mathrm{RF}$ absorption spectra of trapped ions, the level of agreement has been very encouraging.

\section{Acknowledgments}

The authors gratefully acknowledge the financial assistance of the Natural Sciences and Engineering Research Council of Canada, Trent University, and Varian Associates, Incorporated.

\section{References}

1. Dawson, P. H.; Whetten, N. R. J. Vac. Sci. Technol. 1968, 5, 1.

2. Dawson, P. H.; Whetten, N. R. Int. J. Mass Spectrom. Ion Phys. 1974, 14, 339.

3. Bonner, R. F.; March, R.E.; Durup, J. Int. J. Mnss Spectrom. Iom Phys. 1976, 22, 17.

4. Bonner, R. F.; March, R. E. Int. J. Mass Spectrom. Ion Phys. $1977,25,411$.

5. Doran, M. C.; Fulford, J. E.; Hughes, R. J.; Morita, Y; March, R. E. Int. I. Mass Spectrom. Ion Phys. 1980, 33, 139,

6. André, J:; Schermann, J. P. Phys. Lett. 1973, A45, 139.

7. Vedel, F.; André, J.; Vedel, M.; Brincourt, G. Phys. Ret. 1983, A27, 2321.

8. Vedel, F.; André, J. Phys. Rev. 1984, A29, 2098.

9. Vedel, F.; André, J. Int. J. Mass Spectrom. Ion Processes 1985, $65,1$.

10. Louris, J. N.; Cooks, R. G.; Syka, J. E. P.; Kelley, P. E.; Stafford, Ir., G. C.; Todd, I. F. I. Anal. Chem. 1987, 59, 1677.

11. Lunney, M. D. N.; Webb, J. P.; Moore, R. B. J. Appl. Phys. 1989, 65, 2883

12. Lunney, M. D.; Moore, R. B. I.E.E.E. Trans. Magnetics 1991, 27, 4174 .

13. Todd, J. F. J. Mass Spec. Rev. 1991, 10, 3.

14. Pedder, R. E.; Yost, R. A. Proceedings of the 36th Annual ASMS Conference on Mass Spectrometry and Allied Topics; San Francisco, CA; 1988; $\mathrm{p} 632$.

15. Julian, Jr., K. K.; Reiser, H,-P.; Cooks, R. G. Personal communication, 1991.

16. Cooks, R. G.; Glish, G. L.; McLuckey, S. A.; Kaiser, Jr., R. E. Chem. Eng. Nezos 1991, 69, 26.

17. Tucker, D. B.; Hameister, C. H.; Bradshaw, S. C.; Hoekman, D. J.; Weber-Grabau, M. Proceedings of the 36th Annwal ASMS Conference on Mass Spectrometry and Allied Topics; San Francisco, CA; 1988, p 628.

18. Reiser, H.-P.; Kaiser, R. E.; Savickas, P. J.; Cooks, R. G. Inl. J. Mass Spectrom. Ion Processes 1991, 106, 237.

19. Reiser, H.-P.; Julian, Jr., R. K.; Cooks, R. G. Int. J. Mass Spectrom. Ion Processes 1992, 121, 49.

20. Julian, Jr., R. K.; Reiser, H.-P.; Cooks, R. G. Int. J. Mass Spectrom. Ion Processes, in press.

21. Baril, M.; Le, R.; Marchand, P. Int. J. Mass Spectrom. Ion Processes 1990, 98, 87.

22. Wang, Y.; Franzen, J. Int. J. Mass Spectromt. Ion Processes 1992, $112,167$.

23. Wang, Y.; Franzen, J.; Wanczek, K.-P. Int. J. Mass Spectrom. Ion Processes 1993, 124, 125.

24. Vedel, F.; Vedel, M. In Advances in Mass Spectrontetry 11A; $P$. Longevialle, Ed. Heyden and Son: London, 1989; 7244.

25. Rebatel, I. Doctoral thesis, University of Provence; 1993.

26. Vedel, F.; Vedel, M.; March, R. E. Int. I. Mass Spectrom. Ion Processes 1990, 99, 125.

27. Paul, W.; Osberghaus O.; Fischer, E. Forschungsberichte des Wirtschaft und Verkehrministeriums Nordrhein Westfalen, 415 Westdeutscher Verlag, Cologne and Opladen, 1958.

28. Fischer, E. Z. Phys, 1959, 156, 1.

29. Jungmann, K.; Hoffnagle, J.; DeVoe, R. G.; Brewer, R. G. Phys. Rev. 1987, A36, 3451.

30. Kelley. P. E.; Stafford, Jr., G. C.; Stephens, D. R. U.S. Patent 4 
540 884, September 10, 1985; Can. Patent 1207 918, July 15, 1986.

31. March, R. E.; McMahon, A. W.; Londry, F. A.; Alfred, R. L.; Todd, J. F. J.; Vedel, F. Int. I. Mass Spectrom. Ion Processes 1989, 95, 119.

32. March, R. F.; MrMahon, A. W.; Allinson, F. T.; I ondry, F. A.; Alfred, R. L.; Todd, J. F. J.; Vedel, F. Int. J. Mass Spectrom. Ion Processes 1990, 99, 109.

33. March, R. E.; Londry, F. A.; Alfred, R. L.; Todd, J. F. J.; Penman, A. D.; Vedel, F.; Vedel, M. Int. I. Mass Spectrom. Ion Processes 1991, 110, 159.

34. March, R. E.; Weir, M. R.; Tkaczyk, M.; Londry, F. A.; Alfred, R. L.; Franklin, A. M.; Langford, M. L.; Todd, J. F. J. Org. Mass Spectrom., in press.

35. Louris, J. Proceedings of the 40th Ammal ASMS Conference on Mass Spectrometry and Allied Topics, Washington, DC, 1992.

36. March, R. E.; Londry, F. A.; Alfred, R. L. Org. Mass Spectrom. 1992, 27, 1151.

37. Catinella, S.; Traldi, P. Personal communication, 1992.

38. Alfred, R.L.; Londry, F.A.; March, R.E. Int. J. Mass Spectrom. Ion Processes, in press.

39. McLachlan, N. W. Theory and Applications of Mathieu Functions; Clarendon: Oxford, 1947.

40. Press, W. H.; Flannery, B. P.; Teukolsky, S. A.; Vetterling, W. T. Numerical Recipes; New York: Cambridge University Press, 1986; p 563.

41. Lorrain, P.; Corson, D. Electromagnetic Fields and Waves, 2nd ed.; W.H. Freeman: San Francisco, 1970; p 86.

42. Collatz, L. The Numerical Treatment of Differential Lquations; Springer: Berlin, 1960; p 407.

43. Gioumousis, G.; Stevenson, D. P. J. Chem. Phys. 1958, $29,294$.

44. Su, T.; Bowers, M. T. I. Chem. Phys 1973, 58, 3027

45. Knight, R. D. Int. J. Mass Spectrom. Ion Processes 1991, 106, 63.

46. Franzen, J. Int. J. Mass Spectrom. Ion Physics 1983, 51, 127.

\section{Appendix A: The Langevin Collision Theory}

Collisions between an ion and atoms of an inert cooling gas are simulated using the Langevin theory [43]. As an ion and an inert gas atom approach each other, the charged ion induces a electric dipole moment in the neutral, resulting in a net attractive force between the two. The impact parameter, $b$, is defined as the distance of closest approach that the two particles would experience if there was no force of inleraclion between them. There is a certain critical value of the impact parameter such that when $b<b_{0}$, a short-lived complex will be formed and a transfer of energy will occur.

The classical potential at an ion-atom separation $r$ is obtained readily from electrostatic theory as

$$
V(r)=\frac{-q_{j}^{2} \alpha_{e}}{2\left(4 \pi \epsilon_{0}\right)^{2}} \frac{1}{r^{4}}
$$

where $q_{i}$ is the charge on the ion, $\boldsymbol{\alpha}_{e}$ is the electric polarizability of the gas atom, and $\epsilon_{\mathrm{n}}$ is the permittivity of free space.
The relative energy of two particles of reduced mass $\mu$ is given by

$$
E_{r e l}=\frac{p_{\|}^{2}}{2 \mu}+\frac{p_{\perp}^{2}}{2 \mu}+V(r)
$$

where $p_{\|}$and $p_{\perp}$ are components of the relative momentum which are, respectively, parallel and perpendicular to the displacement vector between the two particles. The last two terms in eq A2 comprise an effective radial potential. Expressing $\mathrm{p}_{\downarrow}$ in terms of $b$, $r$, and the total relative kinetic energy of the two particles, $E_{k i n}$, the effective radial potential can be written as

$$
V_{e f f}(r)=E_{k i n} \frac{b^{2}}{r^{2}}-\frac{q_{i}^{2} \alpha_{e}}{2\left(4 \pi \epsilon_{0}\right)^{2}} \frac{1}{r^{4}}
$$

where eq $\mathrm{A} 1$ has been used to expand $V(r) . V_{e f f}(r)$ exhibits a maximum value at $r=r_{0}$. For a capture (energy transfer) to occur, $r$ must be less than $r_{0}$ and the total kinetic energy, $E_{k i n}$ must be sufficiently great to overcome $V_{\text {eff }}\left(r_{0}\right)$. By applying these criteria to eq A3 one can obtain the following expression for the maximum value of the impact parameter for which a capture will occur as

$$
b_{0}=\frac{2 q_{i}}{4 \pi \epsilon_{0} \nu} \sqrt{\frac{\alpha_{e}}{\mu}}
$$

where $v$ is the relative velocity of the collision partners.

Therefore the collision cross-section for a point-particle ion of charge $q$ with an atom or molecule with no permanent dipole moment is given by the Langevin theory [44] as

$$
\sigma=\pi b_{0}^{2}=\frac{2 \pi e}{4 \pi \epsilon_{0} \nu} \sqrt{\frac{\alpha_{e}}{\mu}}
$$

The probability of a collision per unit time can be calculated from the collision cross-section and the number density of collision-gas atoms, $n$, as

$$
P=n \nu \sigma
$$

Using the ideal gas law to approximate $n$ and substituting for $\sigma$ from eq A5,

$$
P=\frac{e}{2 \epsilon_{0}} \sqrt{\frac{\alpha_{\varepsilon}}{\mu}} \frac{p}{k T}
$$

where $p$ and $T$ are the pressure and temperature of the collision-gas, respectively, and $k$ is the Boltzmann constant. Note that $P$ is independent of velocity.

\section{Appendix B: Theory of the Multipole Ion Trap}

From the theory of differential equations, it is well known that a solution of Laplace's equation in spheri- 
cal polar coordinates $(r, \boldsymbol{\theta}, \boldsymbol{\phi})$ for a system with axial symmetry about the $z$-axis has the general form

$$
\Phi(r, \theta, \phi)=\sum_{n=0}^{\infty} A_{n}^{0} r^{n} P_{n}(\cos \theta)
$$

where $A_{n}^{0}$ are arbitrary coefficients, and $P_{n}(\cos \theta)$ denotes a Legendre polynomial. Rewriting eq B1 with $r^{n} P_{n}(\cos \theta)$ expressed in cylindrical polar coordinates $(\rho, \phi, z)$, one obtains

$$
\begin{aligned}
\Phi(\rho, \phi, z)= & A_{0}^{0}+A_{1}^{0} z+A_{2}^{\mathrm{D}}\left(\frac{1}{2} \rho^{2}-z^{2}\right) \\
& +A_{3}^{0} z\left(\frac{3}{2} \rho^{2}-z^{2}\right) \\
& +A_{4}^{0}\left(\frac{3}{8} \rho^{4}-3 \rho^{2} z^{2}+z^{4}\right)+\ldots
\end{aligned}
$$

The terms with $n=0,1,2,3$, and 4 in Eqs. B1 and B2 correspond to the monopole, dipole, quadrupole, hexapole, and octupole components, respectively, of the potential field $\boldsymbol{\Phi}$.

In the case of a multipole ion trap where $\Phi_{0}^{R}$ is the ring potential, $\boldsymbol{\Phi}_{0}^{E}$ the potential on both end-cap electrodes, and $\rho_{0}$ the equatorial radius, one can write the coefficients in eq B2 in a more convenient form as

$$
\begin{gathered}
A_{0}^{0}=B \\
A_{1}^{0}=0 \\
A_{2}^{0}=2 A \\
A_{3}^{0}=2 A\left(A_{3}\right)
\end{gathered}
$$

and

$$
A_{4}^{0}=\frac{8}{3} A\left(A_{4}\right)
$$

Note that $A_{1}^{0}=0$ because there is usually no dipole component in the ion-trap. By using eqs B3-B7, eq B2 can be rewritten as

$$
\begin{aligned}
& \Phi(\rho, z)=A\left[\left(\begin{array}{ll}
\rho^{2} & \left.2 z^{2}\right)
\end{array} \mid A_{3}\left(3 \rho^{2}-2 z^{2}\right) z\right.\right. \\
& \left.+A_{4}\left(\rho^{4}-8 \rho^{2} z^{2}+\frac{8}{3} z^{4}\right)\right]+B
\end{aligned}
$$

where $A_{3}$ and $A_{4}$ are constants related to the multipole field component fractions in the trap and $A$ and $B$ are constants to be determined from the boundary conditions on the trap electrodes.

The trap electrodes must be surfaces which correspond to equipotential surfaces in the trapping field. For a potential field given by eq $\mathrm{B} 8$, the equipotential corresponding to the ring electrode will have the form

$$
\begin{aligned}
& \left(\rho^{2}-2 z^{2}\right)+A_{3}\left(3 \rho^{2}-2 z^{2}\right) z \\
& \quad+A_{4}\left(\rho^{4}-8 \rho^{2} z^{2}+\frac{8}{3} z^{4}\right)=r_{0}^{2} \lambda
\end{aligned}
$$

where $\lambda$ is a positive dimensionless number and $r_{0}$ is an arbitrary displacement. Note that $\lambda$ and $\gamma_{0}$ are not independent parameters. The end-cap electrodes will have the form

$$
\begin{aligned}
& \left(\rho^{2}-2 z^{2}\right)+A_{3}\left(3 \rho^{2}-2 z^{2}\right) z \\
& \quad+A_{4}\left(\rho^{4}-8 \rho^{2} z^{2}+\frac{8}{3} z^{4}\right)=-r_{0}^{2} \mu
\end{aligned}
$$

where $\mu$ is an arbitrary positive dimensionless number. As above, $\mu$ and $r_{0}$ are not independent.

For the electrode surfaces given by eqs B9 and B10, the equatorial radius, $p_{0}$ is given by

$$
\rho_{0}^{2}+A_{4} \rho_{0}^{4}=\lambda r_{0}^{2}
$$

and the polar distance, the displacement of the end-cap electrodes along the line $\rho=0$ from the origin at the center of the trap, $z_{0}$, by

$$
z_{0}^{2}+A_{3} z_{0}^{3}-\frac{4}{3} A_{4} z_{0}^{4}=\frac{1}{2} \mu r_{0}^{2}
$$

Clearly the solution of eq B12 for given values of $\mu$ and $r_{0}$ will yield different magnitudes for positive and negative values of $z_{0}$ when the hexapole field component fraction is nonzero.

From eqs B11 and B12 the ratio of $\mu$ to $\lambda$ can be obtained as

$$
\frac{\mu}{\lambda}=\frac{2 z_{0}^{2}}{\rho_{0}^{2}}\left[\frac{1+A_{3} z_{0}-\frac{4}{3} A_{4} z_{0}^{2}}{1+A_{4} \rho_{0}^{2}}\right]
$$

For a given ratio of $\mu / \lambda$ and given $\rho_{0}$ one can solve eq 13 for $z_{0}$. Because of the $z_{00}^{3}$ term, the solution where $z_{0}$ is positive and that where $z_{0}$ is negative will have different absolute magnitudes. To fix the values of $\mu$ and $\lambda$, the arbitrary parameter $r_{0}$ is assigned the value of the equatorial radius, that is,

$$
r_{0} \equiv \rho_{0}
$$

in the work that follows. Adopting these conventions, fixed values for $\mu$ and $\lambda$ can be calculated from eqs 14 and 15 as

$$
\lambda=1+A_{4} \rho_{0}^{2}
$$

and

$$
\mu=\frac{2 z_{0}^{2}}{\rho_{0}^{2}}\left[1+A_{3} z_{0}-\frac{4}{3} A_{4} z_{0}^{2}\right]
$$

respectively.

It now remains to obtain expressions for the coefficients $A, A_{3}, A_{4}$, and $B$. $A$ and $B$ can be obtained from the boundary conditions on the electrode surfaces. When the potential is $\Phi=\Phi_{0}^{R}$ on the ring electrode, eqs B8, B9, and B14 can be combined to obtain

$$
\Phi(\rho, z)=\Phi\left(\rho_{0}, 0\right)=A\left(\lambda \rho_{0}^{2}\right)+B=\Phi_{0}^{R}
$$

Similarly, when the potential is $\Phi=\boldsymbol{\Phi}_{0}^{E}$ on the end-cap electrodes, eqs B8, B10, and B14 can be combined to 
obtain

$$
\boldsymbol{\Psi}(\rho, z)=\boldsymbol{\Psi}\left(0, z_{0}\right)=A\left(-\mu \rho_{0}^{2}\right)+B=\Psi_{0}^{E}
$$

Solving eqs B17 and B18 for $A$ and $B$, one obtains

$$
A=\frac{\Phi_{0}^{R}-\Phi_{0}^{E}}{(\lambda+\mu) \rho_{0}^{2}}
$$

and

$$
B=\frac{\mu \Phi_{0}^{R}+\lambda \Phi_{0}^{E}}{\lambda+\mu}
$$

respectively. Thus the boundary conditions at the electrode surfaces are always satisfied regardless of the values of the coefficients $A_{3}$ and $A_{4}$.

Substituting eqs B19 and B20 in eq B8 yields

$$
\begin{aligned}
\Phi(\rho, z)= & \frac{\Phi_{0}^{R}-\Phi_{0}^{E}}{(\lambda+\mu) \rho_{0}^{2}}\left[\left(\rho^{2}-2 z^{2}\right)\right. \\
& +A_{3}\left(3 \rho^{2}-2 z^{2}\right) z \\
& \left.+A_{4}\left(\rho^{4}-8 \rho^{2} z^{2}+\frac{8}{3} z^{4}\right)\right]+\frac{\mu \Phi_{0}^{R}+\lambda \Phi_{0}^{E}}{\lambda+\mu}
\end{aligned}
$$

which is the general expression for the field inside an ion trap when the electrodes are surfaces given by eqs B9 and B10 and when the geometric parameters $\rho_{0}$ and $z_{0}$ can be chosen arbitrarily.

When the trap is purely quadrupolar, that is, when the parameters $A_{3}$ and $A_{4}$ which are related to the hexapole and octupole fractions are identically zero, then, using eqs B15 and B16 to replace $\mu$ and $\lambda$, eq B21 simplifies to

$$
\Phi(\rho, z)=\frac{\Phi_{0}^{K}-\Phi_{0}^{E}}{\rho_{0}^{2}+2 z_{0}^{2}}\left[\rho^{2}-2 z^{2}\right]+\frac{2 z_{0}^{2} \Phi_{0}^{R}+\rho_{0}^{2} \Phi_{0}^{L}}{\rho_{0}^{2}+2 z^{2}}
$$

Equation B22 is identical in form to the general expression for the potential inside a quadrupolar ion trap given by Knight [45]. Furthermore, when the ratio of $\rho_{0}^{2}$ to $z_{0}^{2}$ is set equal to 2 in eq B22, then the more commonly used expression

$$
\Phi(p, z)=\frac{\Phi_{0}^{R}-\Phi_{0}^{L}}{2 \rho_{0}^{2}}\left[\rho^{2}-2 z^{2}\right]+\frac{1}{2}\left(\Phi_{0}^{R}+\Phi_{0}^{E}\right)
$$

is obtained.

It now remains to find expressions for the coefficients $A_{3}$ and $A_{4}$ in terms of the multipole field fractions. From electromagnetic theory, the electric field can be obtained from eq $\mathrm{B} 8$ by using the familiar expression $\vec{E}=-\nabla \Phi$, as

$$
E_{p}\left(\rho_{r} z\right)=-2 A\left[\rho+3 A_{3} \rho z+2 A_{4}\left(\rho^{3}-4 \rho z^{2}\right)\right]
$$

and

$$
\begin{aligned}
E_{z}(\rho, z)= & -A\left[-4 z+3 A_{3}\left(\rho^{2}-2 z^{2}\right)\right. \\
& \left.+16 A_{4}\left(-\rho^{2} z+\frac{2}{3} z^{3}\right)\right]
\end{aligned}
$$

From eqs B24 and B25 the net electric field at the ring equator, that is, at the point $\left(\rho_{0}, 0\right)$, can be evaluated as

$$
\left|\vec{E}\left(\rho_{0}, 0\right)\right|=A \rho_{0} \sqrt{4+9 A_{3}^{2} \rho_{0}^{2}+16 A_{4} \rho_{0}^{2}+16 A_{4}^{2} \rho_{0}^{4}}
$$

Similarly, one finds for the hexapole component of the electric field at the ring equator

$$
\left|\vec{E}_{H e x}\left(\rho_{0}, 0\right)\right|=-3 A A_{3} \rho_{0}^{2}
$$

and for the octupole component

$$
\left|\vec{E}_{O C t}\left(\rho_{0}, 0\right)\right|=-3 A A_{4} \rho_{0}^{3}
$$

By using Franzen's definitions of the multipole field component fractions [46] as being the ratio of the field strengths at the ring equator, one obtains from eqs $\mathrm{B} 26-\mathrm{B} 28$ the following expressions:

$$
\begin{aligned}
f_{3} & =\frac{\left|\vec{E}_{H e x}\left(\rho_{0}, 0\right)\right|}{\left|\vec{E}\left(\rho_{0}, 0\right)\right|} \\
& =\frac{3 A_{3} \rho_{0}}{\sqrt{4+9 A_{3}^{2} \rho_{0}^{2}+16 A_{4} \rho_{0}^{2}+16 A_{4}^{2} \rho_{0}^{4}}}
\end{aligned}
$$

and

$$
\begin{aligned}
f_{4} & =\frac{\left|\vec{E}_{O C t}\left(\rho_{0}, 0\right)\right|}{\left|\vec{E}\left(\rho_{0}, 0\right)\right|} \\
& =\frac{4 A_{4} \rho_{0}^{2}}{\sqrt{4+9 A_{3}^{2} \rho_{0}^{2}+16 A_{4} \rho_{0}^{2}+16 A_{4}^{2} \rho_{0}^{4}}}
\end{aligned}
$$

where $f_{3}$ and $f_{4}$ denote the hexapole and octupole field-component fractions, respectively.

By solving eqs $\mathrm{B} 29$ and $\mathrm{B} 30$ for $A_{3}$ and $A_{4}$ it can be shown that

$$
A_{3}=\sqrt{\frac{f_{3}^{2}}{1-f_{3}^{2}}} \frac{1}{1-a} \frac{2}{3 \rho_{0}}
$$


and

$$
A_{4}=\frac{a}{1-a} \frac{1}{2 \rho_{0}^{2}}
$$

where

$$
a=\sqrt{\frac{f_{4}^{2}}{1-f_{3}^{2}}}
$$

It is worth pointing out here that although the multipole fractions $f_{3}$ and $f_{4}$ can be chosen arbitrarily, the coefficients $A_{3}$ and $A_{4}$ depend not only on $f_{3}$ and $f_{4}$ but also on $\rho_{0}$ because $f_{3}$ and $f_{4}$, as defined are determined at $\rho=\rho_{0}$. When $f_{3}$ and $f_{4}$ are small, $a \approx f_{4}$ and

$$
A_{3}=\frac{2 f_{3}}{3 p_{0}}
$$

and

$$
A_{4}=\frac{f_{4}}{2 \rho_{0}^{2}}
$$

From eqs $\mathrm{B} 31$ and $\mathrm{B} 33$ it is readily shown that $A_{3}$ is nonzero only when the hexapole field-component fraction $f_{3}$ is nonzero. Similarly, from eqs B32 and B33, $A_{4}$ is nonzero only when the octupole field-component fraction, $f_{4}$, is nonzern. 